\title{
A new general expression for 2-channel FIR paraunitary filterbanks
}

\author{
M. Elena Domínguez Jiménez
}

Dept. Matemática Aplicada, E.T.S.I. Industriales, Universidad Politécnica de Madrid, C/José Gutiérrez Abascal, 2, 28006 Madrid, Spain

\begin{abstract}
This paper provides a new explicit expression for all real FIR paraunitary filters. From this we derive a new procedure for the design of the $L$ components of any 2-channel FIR orthogonal $L$-tap filter. The input of this algorithm is any set of $L / 2$ free parameters. Moreover, our procedure provides a parameterization of all orthogonal filters directly, with no need for iterations. For the special case of low-pass paraunitary filters, we obtain a fast new design algorithm. Simplified expressions for the frequency response of paraunitary filters are also provided. In addition to these improvements, our results also yield exact solutions of a particular set of Bézout polynomial equations.
\end{abstract}

Keywords: FIR filters; Paraunitary 2-channel filterbanks; Filter design; Bézout equations

\section{Introduction}

Filterbanks play a very important role in many signal processing applications. In particular, for signal compression purposes, the signal is usually first modified by means of a filterbank (or a subband transform) after which some quantization error is introduced in the subband coefficients. By applying the inverse transform to the modified coefficients, we then obtain the compressed signal. But the quantization error is not maintained unless the subband transform is orthogonal; hence, when using filters for this purpose, they must constitute paraunitary filterbanks.

For this reason, many techniques for designing paraunitary filters have been presented in the literature. Wavelet theory has also provided new advances into the filter bank theory, yielding new methods for 2-channel FIR filter design.

In this work, we will focus on 2-channel real causal FIR paraunitary filterbanks. Among the many solutions that can be found in the literature,

They can be divided into two main groups:

(a) Spectral factorization methods: They are based on the fact that a real $L$-tap filter $\mathbf{h}=$ $\left(h_{1}, h_{2}, \ldots, h_{L}\right)$ is paraunitary if and only if its transfer function $H(z)=\sum_{n=1}^{L} h_{n} z^{1-n}$ satisfies

$|H(z)|^{2}+|H(-z)|^{2}=2 \quad \forall|z|=1$.

Hence, it suffices to find the power spectral $P(z)=|H(z)|^{2}$ which verifies $P(z)+P(-z)=2$, and then factor it as $P(z)=|H(z)|^{2}=H(z) H\left(z^{-1}\right)$ in order to get the real filter coefficients. 
Although these methods are commonly used, for instance, in wavelet theory their drawback in the orthogonal case is the need to compute the roots of a $2 L-1$ degree polynomial $P$, which becomes numerically unstable for long filters.

(b) Lattice filters design: This approach designs the polyphase matrix associated with the FIR 2-channel cell given by filters $\mathbf{h}, \mathbf{g}$. The polyphase matrix is defined as

$H_{p}(z)=\left(\begin{array}{cc}H_{\text {even }}(z) & H_{\text {odd }}(z) \\ G_{\text {even }}(z) & G_{\text {odd }}(z)\end{array}\right)$,

where

$$
\begin{aligned}
& H_{\text {even }}(z)=\sum_{n=1}^{L / 2} h_{2 n} z^{1-n} \\
& H_{\text {odd }}(z)=\sum_{n=1}^{L / 2} h_{2 n-1} z^{1-n}
\end{aligned}
$$

and then $H(z)=H_{\text {even }}\left(z^{2}\right)+z^{-1} H_{\text {odd }}\left(z^{2}\right)$. Thus, the filterbank is paraunitary if and only if $H_{p}(z)$ is unitary for every $|z|=1$; hence, it suffices to build unitary matrices of this kind.

The paradigm of these methods is Vaidyanathan's algorithm any paraunitary real $L$-tap FIR filter can be obtained through iteration, by factoring its polyphase matrix as

$H_{p}(z)=\left(\prod_{j=1}^{L / 2-1}\left(I+\left(z^{-1}-1\right) \mathbf{v}_{j} \mathbf{v}_{j}^{\mathrm{t}}\right)\right) Q$,

where $Q$ is unitary of order 2 , and $\mathbf{v}_{j}$ are unitary column vectors of $\mathbb{R}^{2}$. This algorithm behaves well numerically, but it turns out to be difficult when imposing extra desired properties upon the filter.

Also included in this class of solutions is the Lifting method which builds filters of length $L$ with desirable properties by lifting filters of smaller length $L-2$. But, for the orthogonal case this technique can be considered as a particular example of the iterative lattice factorization algorithm already mentioned.

In summary, all the well-known design procedures have their corresponding shortcomings.

Another recent approach makes use of a complete explicit parameterization of all paraunitary 2-channel filterbanks, with no need for factorization or any iterative procedure. Nevertheless, the proposed expression is quite complicated. In this paper we will present a new explicit expression, simpler than any known current solutions.

The paper is organized as follows: in Section 2 we present a new parameterization of all orthogonal filters. In Section 3 we study the particular case of paraunitary filterbanks, and provide a design method for these important filters; illustrative examples are also shown. Besides, by imposing the low-pass condition, we obtain parameterizations for low-pass paraunitary filters. In Section 4 we provide a new and simple expression for the frequency response of such filters. Our final conclusions are presented in Section 5.

Throughout this paper, only real matrices and vectors are considered. Matrices are denoted by capital letters, and vectors by boldface lowercase letters. The superscript $t$ denotes transposition.

\section{General parameterization of orthogonal filters}

Throughout this paper, we will say that a filter $\mathbf{h}$ of even length $L$ is orthogonal if it is orthogonal to its even shifts, that is, if it satisfies

$\forall k=1, \ldots, L / 2-1, \quad 0=\sum_{n=1}^{L-2 k} h_{n} h_{n+2 k}$.

If we additionally impose the norm 1 condition $\left(\sum_{n=1}^{L} h_{n}^{2}=1\right)$, then $\mathbf{h}$ will be called paraunitary.

Now the key question arises: Can we always write the even components of the filter by means of the odd ones, and vice versa? In a previous work we have shown that answer is yes. We reproduce that result here:

Theorem 1. $\mathbf{h}=\left(h_{1}, h_{2}, \ldots, h_{L}\right)$ is an orthogonal filter if and only if there exist $L / 2-1$ real numbers $a_{1}, \ldots, a_{L / 2-1}$ such that, for $k=1, \ldots, L / 2-1$ :

$h_{L+1-2 k}=-\sum_{j=1}^{k} h_{L-2 k+2 j} a_{j}$,

$h_{2 k}=\sum_{j=1}^{k} h_{2 k+1-2 j} a_{j}$.

These equations show that the even components can be generated from the odd ones, and vice versa, by means of $L / 2-1$ free parameters, in addition to $h_{1}$ and $h_{L}$. In other words, the number of degrees of freedom is $L / 2+1$. But, for instance, could we get rid of $h_{L}$ ? Yes, but we would have to include a new parameter $\left(a_{L / 2}\right)$ to maintain the number of free 
parameters. This way, we present the following result, in which an alternative expression is given:

Corollary $\mathbf{1} . \mathbf{h}=\left(h_{1}, h_{2}, \ldots, h_{L}\right)$ is an orthogonal filter if and only if there exist $L / 2$ real numbers $a_{1}, \ldots, a_{L / 2}$ such that

$h_{L+\mathbf{1}-2 k}=-\sum_{j=1}^{k} h_{L-2 k+2 j} a_{j} \quad \forall k=1, \ldots, L / 2-1$,

$h_{2 k}=\sum_{j=1}^{k} h_{2 k+1-2 j} a_{j} \quad \forall k=1, \ldots, L / 2$.

Proof. The first equation is a restatement of Theorem 1. As for the second equation, we just have to define $a_{L / 2}$ such that

$a_{L / 2} h_{1}+a_{L / 2-1} h_{3}+\cdots+a_{1} h_{L-1}=h_{L}$.

Since $h_{1} \neq 0$ by definition, then we can always define

$a_{L / 2}=\frac{h_{L}-a_{L / 2-1} h_{3}+\cdots+a_{1} h_{L-1}}{h_{1}}$

so Eq. (5) is guaranteed.

\subsection{Generation of orthogonal filters}

Now we will obtain a new explicit expression for all orthogonal filters. Notice that the two identities of Corollary 1 present some kind of redundancy: the coefficient $h_{L}$ appears as a parameter in Eq. (4) and as an unknown in (5). Can we exploit this redundancy? In other words, can we generate $\mathbf{h}$ using only external independent parameters, such as $a_{1}, \ldots, a_{L / 2}$ ? We will show that the answer is yes, whenever we fix the first nonzero coefficient $h_{1}$. This will then lead us to find the desired design method for all orthogonal filters.

To this end, by means of the parameters $\left(a_{1}, \ldots, a_{L / 2}\right)$, we define:

- a Toeplitz low triangular matrix of order $L / 2-1$ :

$$
A=\left(\begin{array}{ccccc}
a_{1} & 0 & 0 & \cdots & 0 \\
a_{2} & a_{1} & 0 & \ddots & \vdots \\
a_{3} & a_{2} & a_{1} & \ddots & \vdots \\
\vdots & \ddots & \ddots & \ddots & 0 \\
a_{L / 2-1} & \cdots & a_{3} & a_{2} & a_{1}
\end{array}\right)
$$

- and three column vectors of length $L / 2-1$ :

$$
\left.\begin{array}{l}
\mathbf{a}=\left(a_{2}, a_{3}, \ldots, a_{L / 2}\right)^{\mathrm{t}} \\
\mathbf{b}=\left(I+A A^{\mathrm{t}}\right)^{-1} \mathbf{a}, \\
\mathbf{c}=-A^{\mathrm{t}} \mathbf{b},
\end{array}\right\}
$$

where $\mathbf{b}$ is well defined because $I+A A^{\mathrm{t}}$ is always a positive definite matrix.

For the sake of simplicity, from now on we will denote the vectors of length $L / 2-1$ :

$$
\begin{aligned}
& \mathbf{h}_{\text {even }}=\left(h_{4}, h_{6}, \ldots, h_{L-2}, h_{L}\right)^{\mathrm{t}}, \\
& \mathbf{h}_{\text {odd }}=\left(h_{3}, h_{5}, \ldots, h_{L-3}, h_{L-1}\right)^{\mathrm{t}},
\end{aligned}
$$

which contain the even and odd indexed coefficients of $\mathbf{h}$ except for $h_{1}, h_{2}$.

Now we are finally ready to express all the components of the filter by means of $h_{1}$ and the $L / 2$ parameters. This is one of the main results of this paper, which constitutes a new parameterization of all orthogonal filters, simpler than the one given in

Theorem 2. $\mathbf{h}=\left(h_{1}, h_{2}, \ldots, h_{L}\right)$ is an orthogonal filter if and only if there exist $L / 2$ real numbers $a_{1}, \ldots, a_{L / 2}$ such that

$$
\left.\begin{array}{l}
h_{2}=h_{1} a_{1}, \\
\mathbf{h}_{\text {even }}=h_{1} \mathbf{b} \\
\mathbf{h}_{\text {odd }}=h_{1} \mathbf{c} .
\end{array}\right\}
$$

Proof. We start by writing Eq. (4) as

$$
\begin{aligned}
\left(\begin{array}{c}
h_{2} \\
\mathbf{h}_{\mathrm{even}}
\end{array}\right) & =\left(\begin{array}{c}
h_{1} a_{1} \\
h_{1} \mathbf{a}
\end{array}\right)+\left(\begin{array}{c}
0 \\
A \mathbf{h}_{\text {odd }}
\end{array}\right) \\
& \Leftrightarrow\left\{\begin{array}{l}
h_{2}=a_{1} h_{1}, \\
\mathbf{h}_{\text {even }}=h_{1} \mathbf{a}+A \mathbf{h}_{\text {odd }}
\end{array}\right.
\end{aligned}
$$

and Eq. (5) as

$\mathbf{h}_{\text {odd }}=-A^{\mathrm{t}} \mathbf{h}_{\text {even }}$

so it suffices to substitute (8) into (7):

$$
\begin{aligned}
\mathbf{h}_{\text {even }} & =h_{1} \mathbf{a}-A A^{\mathrm{t}} \mathbf{h}_{\text {even }} \\
& \Leftrightarrow\left(I+A A^{\mathrm{t}}\right) \mathbf{h}_{\text {even }}=h_{1} \mathbf{a} \\
& \Leftrightarrow \mathbf{h}_{\text {even }}=h_{\mathbf{1}}\left(I+A A^{\mathrm{t}}\right)^{-\mathbf{1}} \mathbf{a}=h_{\mathbf{1}} \mathbf{b} .
\end{aligned}
$$

We just finally have

$\mathbf{h}_{\text {odd }}=-A^{\mathrm{t}} \mathbf{h}_{\text {even }}=-h_{1} A^{\mathrm{t}} \mathbf{b}=h_{1} \mathbf{c}$.

We have now derived that, by choosing $L / 2$ arbitrary parameters and one arbitrary nonzero 
number $h_{1}$, the whole set of orthogonal filters $\mathbf{h}$ of length $L$ can be parameterized. In other words, these filters are characterized by means of just $L / 2+1$ parameters. And this representation is unique: different sets of parameters always yield different filters, so there is no redundancy in this parameterization.

The simplified general expression for orthogonal filters we have obtained is

$$
\left(\begin{array}{c}
h_{1} \\
h_{\text {odd }} \\
h_{2} \\
h_{\text {even }}
\end{array}\right)=h_{1}\left(\begin{array}{c}
1 \\
\mathbf{c} \\
a_{1} \\
\mathbf{b}
\end{array}\right) \text {. }
$$

The filter is parameterized by choosing just $L / 2$ free parameters $a_{1}, \ldots, a_{L / 2}$ (which are used to compute b and c) plus its first coefficient $h_{1}$.

Then, the even and odd components have the following impulse responses:

$$
\left.\begin{array}{l}
H_{\text {even }}(z)=\sum_{k=0}^{L / 2-1} h_{2 k+2} z^{-k}=h_{1} B\left(z^{-1}\right), \\
H_{\text {odd }}(z)=\sum_{k=0}^{L / 2-1} h_{2 k+1} z^{-k}=h_{1} C\left(z^{-1}\right),
\end{array}\right\}
$$

where the polynomials $B$ and $C$ are

$$
B(z)=a_{1}+\sum_{k=1}^{L / 2-1} b_{k} z^{k}, \quad C(z)=1+\sum_{k=1}^{L / 2-1} c_{k} z^{k} .
$$

In other words, $B\left(z^{-1}\right), C\left(z^{-1}\right)$ are the transfer functions of the filters $\left(a_{1}, \mathbf{b}\right)$ and $(1, \mathbf{c})$, respectively. They yield the even and odd components of the filter $\mathbf{h}$, up to the factor $h_{1}$.

Therefore, the impulse response of the orthogonal filter is

$$
\begin{aligned}
H(z) & =H_{\text {odd }}\left(z^{2}\right)+z^{-1} H_{\text {even }}\left(z^{2}\right) \\
& =h_{1}\left(C\left(z^{-2}\right)+z^{-1} B\left(z^{-2}\right)\right) .
\end{aligned}
$$

\section{General expression for paraunitary filters}

In this section we will add extra properties to the filter. First, we impose it to be paraunitary, that is, the vector $\mathbf{h}$ is unitary. This is the same as asserting that the vector of Eq. (9) has norm 1:

$$
1=h_{1}^{2}\left(1+a_{1}^{2}+\|\mathbf{c}\|^{2}+\|\mathbf{b}\|^{2}\right)
$$

and so, this condition determines $h_{1}$ (up to its sign). By using the relationship between $\mathbf{b}$ and $\mathbf{c}$ we can simplify this expression:

$$
\begin{aligned}
\|\mathbf{c}\|^{2}+\|\mathbf{b}\|^{2} & =\mathbf{c}^{\mathrm{t}} \mathbf{c}+\mathbf{b}^{\mathrm{t}} \mathbf{b}=\mathbf{b}^{\mathrm{t}} A A^{\mathrm{t}} \mathbf{b}+\mathbf{b}^{\mathrm{t}} \mathbf{b} \\
& =\mathbf{b}^{\mathrm{t}}\left(A A^{\mathrm{t}}+I\right) \mathbf{b}=\mathbf{b}^{\mathrm{t}} \mathbf{a} \geqslant 0
\end{aligned}
$$

so the unitary condition is

$h_{1}^{2}=\frac{1}{1+a_{1}^{2}+\mathbf{b}^{\mathbf{t}} \mathbf{a}}$.

Thus, we obtain the complete set of paraunitary filters of length $L$ :

Corollary 2 . $\mathbf{h}$ is a paraunitary filter if and only if its components can be written as

$$
\left(\begin{array}{c}
h_{1} \\
h_{\mathrm{odd}} \\
h_{2} \\
h_{\mathrm{even}}
\end{array}\right)=\frac{ \pm 1}{\sqrt{1+a_{1}^{2}+\mathbf{b}^{\mathrm{t}} \mathbf{a}}}\left(\begin{array}{c}
1 \\
\mathbf{c} \\
a_{1} \\
\mathbf{b}
\end{array}\right) .
$$

Notice that these filters are expressed directly via $L / 2$ parameters $a_{1}, \ldots, a_{L / 2}$ and one sign. This is the simplest general expression for paraunitary filters that we have obtained, and this constitutes one of the main results of this paper.

Now, it is well known that a filter is paraunitary if and only if

$\forall|z|=1, \quad\left|H_{\text {odd }}(z)\right|^{2}+\left|H_{\text {even }}(z)\right|^{2}=1$,

in effect: recall that the polyphase matrix of Eq. (2) is unitary. So, using Eqs. (10) and (12), we arrive at the following result:

$$
\begin{aligned}
& \forall|z|=1, \\
& \begin{aligned}
|B(z)|^{2}+|C(z)|^{2} & =\frac{1}{h_{1}^{2}} \\
& =1+\|\mathbf{c}\|^{2}+a_{1}^{2}+\|\mathbf{b}\|^{2} .
\end{aligned}
\end{aligned}
$$

Moreover, recalling the expression (11) for polynomials $B$ and $C$, we notice that this last term equals $\|B\|^{2}+\|C\|^{2}$, where $\|B\|$ denotes the Euclidean norm of the vector which contains its coefficients $\left(a_{1}, \mathbf{b}^{\dagger}\right)$, and where $\|C\|$ denotes the Euclidean norm of its corresponding coefficients vector $\left(1, \mathbf{c}^{\mathrm{t}}\right)$.

The last equation is very important, because we now know that polynomial equations of this type can be solved by means of our design approach:

Theorem 3. Let $B(z)$ and $C(z)$ be real polynomials, and let $\|B\|,\|C\|$ denote the Euclidean norm of the 
vectors which contain their respective coefficients. Then, the following statements are equivalent:

(1) $B, C$ satisfy the equation

$$
|B(z)|^{2}+|C(z)|^{2}=\|B\|^{2}+\|C\|^{2} \quad \forall|z|=1 .
$$

(2) The coefficients of $B$ and $C$ are, respectively, the even and odd coefficients of an orthogonal filter $\mathbf{h}$.

(3) The coefficients of

$$
\frac{B}{\sqrt{\|B\|^{2}+\|C\|^{2}}}, \quad \frac{C}{\sqrt{\|B\|^{2}+\|C\|^{2}}}
$$

are, respectively, the even and odd coefficients of a paraunitary filter $\mathbf{h}$.

(4) $B, C$ can be explicitly computed via our design method, using Eqs. (11) and (6).

This important result gives a new characterization of all solutions of the Bézout polynomial equations on the unit circle

$|B(z)|^{2}+|C(z)|^{2}=\|B\|^{2}+\|C\|^{2} \quad \forall|z|=1$.

These particular Bézout identities not only appear in filter design, but also in other mathematical problems. This exact solution thus constitutes one of the most important results of this paper.

\subsection{Generation of low-pass paraunitary filters}

We will now impose the low-pass constraint so as to find the general expression for all low-pass paraunitary filters. It is well known that in this case $H(-1)=0$; besides, as the filter is paraunitary, the orthogonality condition (1) is fulfilled. By taking $z=1$, then $H(1)=\sqrt{2}$, which is equivalent to the following condition:

$H_{\text {even }}(1)=H_{\text {odd }}(1)$.

Since we have the expression

$H_{\text {odd }}(1)=h_{1} C(1), \quad H_{\text {even }}(1)=h_{1} B(1)$

we arrive at the condition

$C(1)=B(1)$

This equality can be used to determine the value of one of the $L / 2$ free parameters, by means of the remaining ones.
Note that we have also obtained the identity

$$
\begin{aligned}
|B(1)|^{2}+|C(1)|^{2} & =\frac{1}{h_{1}^{2}}=1+a_{1}^{2}+\mathbf{b}^{\mathrm{t}} \mathbf{a}=2|C(1)|^{2} \\
& =2 B(1) C(1) .
\end{aligned}
$$

\subsection{Example: 4-tap low-pass paraunitary filters}

In this case, $L=4$ and the general expression for the odd and even components of any orthogonal filter of length 4 is

$$
\left(\begin{array}{l}
h_{1} \\
h_{3} \\
h_{2} \\
h_{4}
\end{array}\right)=h_{1}\left(\begin{array}{c}
1 \\
c \\
a_{1} \\
b
\end{array}\right) \text {, }
$$

where there are $L / 2=2$ free parameters: $a_{1}$ and $h_{1}$. Note that $\mathbf{b}, \mathbf{c}$ are vectors of length $L / 2-1=1$, so they are scalars, as well as the matrix $A=a_{1}$ :

$$
\begin{aligned}
& \mathbf{b}=\left(1+a_{1}^{2}\right)^{-1} a_{2}, \\
& \mathbf{c}=-A^{\mathrm{t}} b=-a_{1} b=-\left(1+a_{1}^{2}\right)^{-1} a_{1} a_{2}
\end{aligned}
$$

so the general orthogonal filter is

$$
\begin{aligned}
\mathbf{h} & =\left(h_{1}, h_{3}, h_{2}, h_{4}\right) \\
& =h_{1}\left(1, a_{1}, c, b\right) \\
& =h_{1}\left(1, a_{1},-\frac{a_{1} a_{2}}{1+a_{1}^{2}}, \frac{a_{2}}{1+a_{1}^{2}}\right) .
\end{aligned}
$$

In case of unitary vectors, after normalizing we obtain the general expression

$$
\mathbf{h}= \pm \frac{\left(1+a_{1}^{2},\left(1+a_{1}^{2}\right) a_{1},-a_{1} a_{2}, a_{2}\right)}{\sqrt{\left(a_{1}^{2}+1\right)\left(a_{2}^{2}+\left(a_{1}^{2}+1\right)^{2}\right)}}
$$

Now, if we impose the low-pass property (16):

$1+c=a_{1}+b$

then

$1-a_{1}=b-c=\left(1+a_{1}\right) b=\left(1+a_{1}\right)\left(1+a_{1}^{2}\right)^{-1} a_{2}$

so it determines $a_{2}$ whenever $a_{1} \neq-1$; in this case,

$a_{2}=\frac{\left(1-a_{1}\right)\left(1+a_{1}^{2}\right)}{\left(1+a_{1}\right)}$.

By introducing it into the general paraunitary 4-tap filter expression (18), we finally achieve the general expression for low-pass paraunitary filters of length 4 :

$\mathbf{h}=\frac{1}{\sqrt{2}\left(1+a_{1}^{2}\right)}\left(1+a_{1}, a_{1}^{2}+a_{1}, a_{1}^{2}-a_{1}, 1-a_{1}\right)$. 
Notice that this general expression is also valid for $a_{1}=-1$; in this case it turns out be the shifted Haar filter:

$\mathbf{h}=\frac{1}{\sqrt{2}}\left(\begin{array}{llll}0 & 0 & 1 & 1\end{array}\right)$,

whereas for $a_{1}=1$ it is Haar filter of length 2:

$\mathbf{h}=\frac{1}{\sqrt{2}}\left(\begin{array}{llll}1 & 1 & 0 & 0\end{array}\right)$.

This result brings no surprises, since any parameterization of paraunitary filters of length 4 must include these filters of length 2 .

Let us compare this technique with the other approaches for designing 4-tap low-pass paraunitary filters: the spectral method would have required a greater amount of operations; on the other hand, using lattice filters we would only need $\frac{4}{2}-1=1$ unitary vector, and a unitary matrix of order 2 . It is easy to see that the unitary matrix for low-pass filters is always equal to

$Q=\frac{\sqrt{2}}{2}\left(\begin{array}{cc}1 & 1 \\ 1 & -1\end{array}\right)$.

Next, by choosing an arbitrary unitary vector $\mathbf{v}=$ $(c, d)^{t} / \sqrt{c^{2}+d^{2}}$ and the unitary matrix $Q$, the paraunitary low-pass filters computed via the lattice method are

$\mathbf{h}=\frac{1}{\left(c^{2}+d^{2}\right) \sqrt{2}}\left(d^{2}-c d, d^{2}+c d, c^{2}+c d, c^{2}-c d\right)$.

Clearly, this expression is more complicated than our result (19).

\section{Power spectral response of paraunitary filters}

Finally, we will obtain a general expression for the power spectral response of paraunitary filters. The impulse response is

$H(z)=H_{\text {odd }}\left(z^{2}\right)+z^{-1} H_{\text {even }}\left(z^{2}\right)$,

and its frequency response is $P(z)=|H(z)|^{2}$. Recalling Eq. (15), the power spectral response of the filter is

$$
\begin{aligned}
P(z) & =|H(z)|^{2}=\left|H_{\text {odd }}\left(z^{2}\right)+z^{-1} H_{\text {even }}\left(z^{2}\right)\right|^{2} \\
& =1+2 \operatorname{Re}\left(z H_{\text {even }}\left(z^{-2}\right) H_{\text {odd }}\left(z^{2}\right)\right) .
\end{aligned}
$$

Now we use the general expression for $H_{\text {even }}, H_{\text {odd }}$ obtained in Eq. (10) for orthogonal filters:

$H_{\text {even }}(z)=h_{1} B\left(z^{-1}\right), \quad H_{\text {odd }}(z)=h_{1} C\left(z^{-1}\right)$.
Since the orthogonality condition (15) is fulfilled, the frequency response of the orthogonal filter is

$P(z)=1+2 h_{1}^{2} \operatorname{Re}\left(z B\left(z^{2}\right) C\left(z^{-2}\right)\right)$.

Therefore, if the filter is paraunitary, by using Eq. (13) we arrive at the following result:

Theorem 4. The power spectral response of any paraunitary filter is

$P(z)=1+\frac{2 \operatorname{Re}\left(z B\left(z^{2}\right) C\left(z^{-2}\right)\right)}{1+a_{1}^{2}+\mathbf{b}^{\mathrm{t}} \mathbf{a}}$.

Note that the coefficients of the polynomial $P$ are the correlation coefficients of the vectors $h_{1}\left(a_{1}, \mathbf{b}^{\mathrm{t}}\right)$ and $h_{1}\left(1, \mathbf{c}^{\mathrm{t}}\right)$. Recall that everything is directly determined from the $L / 2$ independent parameters $a_{1}, \ldots, a_{L / 2}$.

Remark 1. Clearly, Eq. (20) represents one of the main results of this paper. It is an explicit, simple, general expression for the power spectral response $P(z)$ of a paraunitary $L$-tap filter, by means of $L / 2$ arbitrary parameters.

Next, it is helpful to rewrite Eq. (20) to obtain a new result: the general expression for the power spectral response of a paraunitary filter is

$P(z)=1+\frac{2 \operatorname{Re}\left(z B\left(z^{2}\right) C\left(z^{-2}\right)\right)}{\left|B\left(z^{2}\right)\right|^{2}+\left|C\left(z^{2}\right)\right|^{2}}$,

where $B(z), C(z)$ are the polynomials obtained via the design method presented in Section 2.

Let us show that, for any arbitrary nonnull polynomials $B(z), C(z), \mathrm{Eq}$. (21) also provides the power spectral response of a paraunitary filter. In effect, $P(z)$ is a halfband filter, and the fact that

$$
\begin{aligned}
2\left|\operatorname{Re}\left(z B\left(z^{2}\right) C\left(z^{-2}\right)\right)\right| & \leqslant 2\left|B\left(z^{2}\right)\right|\left|C\left(z^{2}\right)\right| \\
& \leqslant\left|B\left(z^{2}\right)\right|^{2}+\left|C\left(z^{2}\right)\right|^{2}
\end{aligned}
$$

assures that $0 \leqslant P(z) \leqslant 2$. Besides, the inequalities become equalities for those $\left|z_{0}\right|=1$ such that $C\left(z_{0}^{2}\right)= \pm z_{0} B\left(z_{0}^{2}\right)$.

Remark 2. We deduce that Eq. (21) is the most general expression for the power spectral response $P(z)$ of paraunitary filters, since it is valid for any pair of nonzero arbitrary polynomials $B, C$. Moreover, if $B, C$ are chosen using our design method, the great advantage is the fact that the denominator is constant:

$$
|B(z)|^{2}+|C(z)|^{2}=1+a_{1}^{2}+\mathbf{b}^{\mathrm{t}} \mathbf{a} .
$$


Therefore, Eq. (20) is the most simplified general expression found to date.

Moving on to low-pass filters, we provide an additional result:

Theorem 5. A paraunitary filter is low-pass if and only if the associated polynomials $B, C$ satisfy $B(1)=C(1)$, or, equivalently,

$\frac{1+a_{1}^{2}+\mathbf{b}^{\mathrm{t}} \mathbf{a}}{2}=B(1) C(1)$.

In this case, the most general expression for the power spectral response of a paraunitary low-pass filter is

$P(z)=1+\frac{\operatorname{Re}\left(z B\left(z^{2}\right) C\left(z^{-2}\right)\right)}{B(1) C(1)}$.

Proof. Starting with the necessary low-pass condition (17), we introduce it in Eq. (20). Moreover, this condition becomes sufficient, because we also derive that $P(1)=2$. Note that for the low-pass filters case, there are $L / 2-1$ degrees of freedom, because the condition of this theorem yields a restriction from which allows us to fix, for instance, the value of $a_{L / 2}$.

Remark 3. This constitutes one of the important results of this paper. The key idea is that the construction of $B$ and $C$ can now easily be carried out using the procedure proposed in Section 2. In order to design the frequency response $P(z)$ associated with a paraunitary low-pass filter, we only need to calculate vectors $\mathbf{b}$ and $\mathbf{c}$, impose Eq. (16), and finally introduce them into Eq. (22).

\subsection{Example: frequency response of 4-tap paraunitary low-pass filters}

For 4-tap paraunitary filters, vectors $\mathbf{a}, \mathbf{b}, \mathbf{c}$ are numbers, so we get

$$
\begin{aligned}
P(z) & =1+\frac{2 \operatorname{Re}\left(z\left(a_{1}+z^{2} b\right)\left(1+z^{-2} c\right)\right)}{1+a_{1}^{2}+b a_{2}} \\
& =1+\frac{2 \operatorname{Re}\left(z^{-1} a_{1} c+z\left(a_{1}+b c\right)+z^{3} b\right)}{1+a_{1}^{2}+b a_{2}}
\end{aligned}
$$

where we know that

$$
b=\frac{a_{2}}{1+a_{1}^{2}}, \quad c=-\frac{a_{1} a_{2}}{1+a_{1}^{2}} .
$$

Since the parameters are real, we have that, for $z=\mathrm{e}^{\mathrm{i} w}$,

$P\left(\mathrm{e}^{\mathrm{i} w}\right)=1+2 \frac{\left(a_{1}(c+1)+b c\right) \cos w+b \cos 3 w}{1+a_{1}^{2}+b a_{2}}$.

Moreover, for low-pass paraunitary 4-tap filters we have the frequency response

$P\left(\mathrm{e}^{\mathrm{i} w}\right)=1+\frac{a_{1}^{2}\left(a_{1}^{2}+3\right)}{\left(1+a_{1}^{2}\right)^{2}} \cos w+\frac{\left(1-a_{1}^{2}\right)}{\left(1+a_{1}^{2}\right)^{2}} \cos 3 w$.

This expression will help us to design filters with certain extra desired properties. For instance, the paraunitary low-pass filters with maximum attenuation in the interval $[\pi / 2, \pi]$ are the ones that minimize

$$
\begin{aligned}
\int_{\pi / 2}^{\pi}\left|H\left(\mathrm{e}^{\mathrm{i} w}\right)\right|^{2} \mathrm{~d} w & =\int_{\pi / 2}^{\pi} P\left(\mathrm{e}^{\mathrm{i} w}\right) \mathrm{d} w \\
& =1-\frac{a_{1}^{2}\left(a_{1}^{2}+3\right)}{\left(1+a_{1}^{2}\right)^{2}}+\frac{\left(1-a_{1}^{2}\right)}{3\left(1+a_{1}^{2}\right)^{2}} .
\end{aligned}
$$

We thus find that the minimum is achieved whenever $a_{1}^{2}=3$. In other words, the optimal frequency response is obtained for

$P\left(\mathrm{e}^{\mathrm{i} w}\right)=1+\frac{9}{8} \cos w-\frac{1}{8} \cos 3 w$.

In order to get the associated low-pass 4-tap filters, we use Eq. (19); for $a_{1}=\sqrt{3}$,

$\mathbf{h}=\frac{1}{4 \sqrt{2}}(1+\sqrt{3}, 3+\sqrt{3}, 3-\sqrt{3}, 1-\sqrt{3})$,

whereas for $a_{1}=-\sqrt{3}$ we obtain

$\mathbf{h}=\frac{1}{4 \sqrt{2}}(1-\sqrt{3}, 3-\sqrt{3}, 3+\sqrt{3}, 1+\sqrt{3})$,

which correspond to the 4-tap Daubechies filters (minimum/maximum phase), whose attenuation is

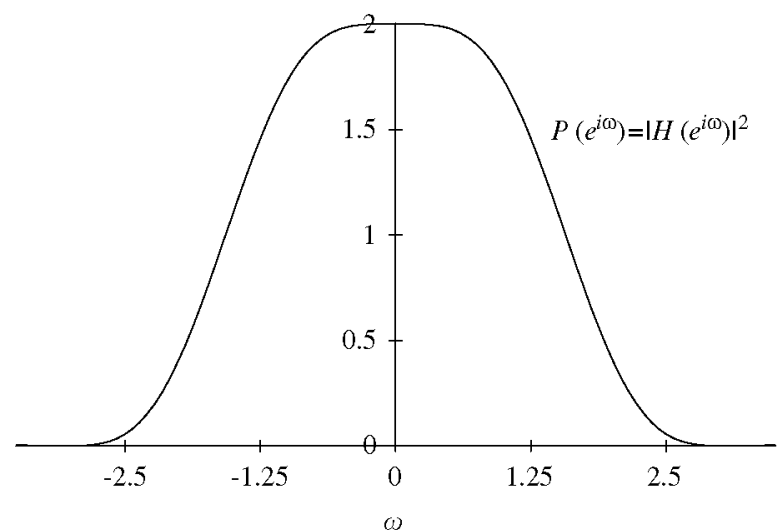

Fig. 1. Maximum attenuation of paraunitary low-pass 4-tap filters. 
optimal. In fact, their power spectral responses $P\left(\mathrm{e}^{\mathrm{i} w}\right)=\left|H\left(\mathrm{e}^{\mathrm{i} w}\right)\right|^{2}$ coincide; it is depicted in Fig. 1.

Let us remark that our technique confirms more directly the results obtained using other approaches. Nevertheless, working with longer filters will involve maximizing a functional which depends on more variables, and the expressions will be more complicated.

\section{Conclusions}

We have presented a new parameterization of real FIR orthogonal filters. The proposed procedure does not need any iteration process; additionally, the direct expression we provide is simpler than other existing ones. We have also obtained the general parameterization of low-pass paraunitary filters. Another important result is an improved, simple, explicit expression for the frequency response of any paraunitary filter. This yields a new powerful tool for designing paraunitary filters which satisfy extra conditions usually required in specific applications. Our work has also produced an additional result: We have found the general solution of a class of Bézout polynomial equations, which appear not only in filter design theory, but also in other disciplines.

\section{Acknowledgements}

This work has been developed through UPM Research Group TACA (Teoría de Aproximación
Constructiva y Aplicaciones) and supported by UPM through the Research Project TITAC, Ref. CCG06-UPM/MTM-539, and by CICYT through the Research Project IN-VENTO, Ref. CGL2005-06966-C07/CLI.

\section{References}

I. Daubechies, Ten Lectures on Wavelets, SIAM, Philadelphia, PA, 1992.

P.P. Vaidyanathan, Multirate Systems and Filter Banks, Prentice-Hall, Englewood Cliffs, NJ, 1993.

M. Vetterli, J. Kovacevic, Wavelets and Subband Coding, Prentice-Hall, Englewood Cliffs, NJ, 1995.

G. Strang, T. Nguyen, Wavelets and Filter Banks, WellesleyCambridge Press, 1996.

I. Daubechies, W. Sweldens, Factoring wavelet transforms into lifting steps, J. Fourier Anal. Appl. 4 (March 1998) 247-269.

C.W. Kim, R. Ansari, A.E. Cetin, A class of linear-phase regular biorthogonal wavelets, in: Proceedings of International Conference on Acoustics, Speech, and Signal Processing (ICASSP-92), vol. 4, March 1992, pp. 673-677.

P.P. Vaidyanathan, Improved technique for design of perfect reconstruction FIR QMF banks with lossless polyphase matrices, IEEE Trans. ASSP 37 (July 1989) 1042-1056.

S.M. Phoong, C.W. Kim, P.P. Vaidyanathan, R. Ansari, A new class of two-channel biorthogonal filter banks and wavelet bases, IEEE Trans. ASSP 43 (March 1995) 649-665. J. Kovacevic, W. Sweldens, Wavelet families of increasing order in arbitrary dimensions, IEEE Trans. Image Process. 9 (March 2000) 480-496.

M.E. Dominguez, Noniterative design of 2-channel FIR orthogonal filters, EURASIP J. Adv. Signal Process. 2007 (January 2007) Article ID 45816. doi:10.1155/2007/45816. 